\title{
Exposing optical near fields of plasmonic patch nanoantennas
}

\author{
Manoj Manjare, ${ }^{1}$ Feng Wang, ${ }^{1}$ Sergio G. Rodrigo, ${ }^{2,3}$ and Hayk Harutyunyan ${ }^{1, a)}$ \\ ${ }^{1}$ Department of Physics, Emory University, Atlanta, Georgia 30322, USA \\ ${ }^{2}$ Centro Universitario de la Defensa, Ctra. de Huesca s/n, E50090 Zaragoza, Spain \\ ${ }^{3}$ Instituto de Ciencia de Materiales de Aragón and Departamento de Física de la Materia Condensada, \\ CSIC-Universidad de Zaragoza, E50009 Zaragoza, Spain
}

(Received 22 August 2017; accepted 11 November 2017; published online 29 November 2017)

\begin{abstract}
Plasmonic nanosystems are typically used in optical experiments to concentrate electromagnetic energy into sub-wavelength volumes. In this context, metal-dielectric-metal (MDM) nanosystems provide an attractive geometry for achieving strong field enhancement and mode confinement on a few-nanometer scale. Unfortunately, the disadvantage of the MDM configuration can be that the enhanced near fields are confined to the dielectric spacer and are not externally accessible. This limits the suitability of such platforms for applications, where the interaction of light with external nano-objects is desirable, such as sensing, detection, and quantum optics. To expose the enhanced near fields to the environment, we selectively and isotropically etch the gap dielectric layer, which results in patch antennas on thin silicon oxide pedestals. We perform optical sensing experiments and simulations and confirm the sensitivity of the optical near fields to the refraction index change in a small volume. Published by AIP Publishing. https://doi.org/10.1063/1.5001199
\end{abstract}

Optically induced collective oscillations of surface electrons in metallic nanosystems give rise to localized resonances called plasmons. These electromagnetic modes are not subject to the same diffraction limits as pure photonic modes and thus can be confined to volumes much smaller than the diffraction limit of free space light. ${ }^{1}$ This ability to focus optical energy into nanometer-scale volumes enables optical experiments and platforms where the light concentration is beneficial, such as nonlinear optics, quantum optics, and optical sensing. ${ }^{2-5}$ For many of these applications, the confinement of optical fields is an important parameter as it can lead to a larger field enhancement and a stronger light-matter interaction.

In isolated nanoparticles, the mode confinement is achieved by exciting decaying evanescent near fields which in the optical regime can be on the order of tens of nanometers [Fig. 1(a)]. These isolated systems typically feature modest field enhancement. To increase the field enhancement even further, multiple interacting elements can be used where charge accumulation in the nanometric gaps between the nanoparticles can concentrate the optical fields. ${ }^{6}$ The field enhancement in these systems scales inversely with the size of the gap and is ultimately limited by nonlocal and quantum tunneling effects. $^{7-9}$ Unfortunately, in top down fabrication techniques such as electron beam lithography (EBL), the nanoparticle gaps that can be reproducibly achieved are on the order of $>20 \mathrm{~nm}$.

Optical patch antennas (or nanoparticles on a mirror) have recently gained popularity as platforms with fewnanometer gaps and strong field confinement. ${ }^{10-14}$ In these systems, nanoparticles are fabricated on top of a smooth metal film separated by a thin dielectric gap [Fig. 1(b)]. Illumination of these metal-dielectric-metal (MDM) systems with far field optical fields excites the localized plasmon modes in the nanoparticles. The oscillating charges of these

a)hayk.harutyunyan@emory.edu plasmonic modes in turn excite mirror charges in the metal film. Attractive coupling between plasmonic charges and their image charges gives rise to strongly enhanced optical fields in the gaps defined by the spacer. Since the thickness of the dielectric layers of the spacer can be controlled with atomic precision, very small nanometer-scale gaps can be easily achieved. The highly confined fields excited in these gaps have been used to enhance nonlinear effects and emission properties of quantum emitters. ${ }^{15-20}$

However, one drawback of the patch antenna geometry is that the near-fields of such structures are confined to the spacer dielectric and are not externally accessible. This can lead to limited performance of the MDM-based systems for, e.g., sensing, particle detection, and plasmon-quantum emitter coupling applications. To expose the near-fields to the environment, in this work, we have selectively and isotropically etched the gap dielectric in MDM nanostructures. To test the exposure of the confined fields in the nanometric
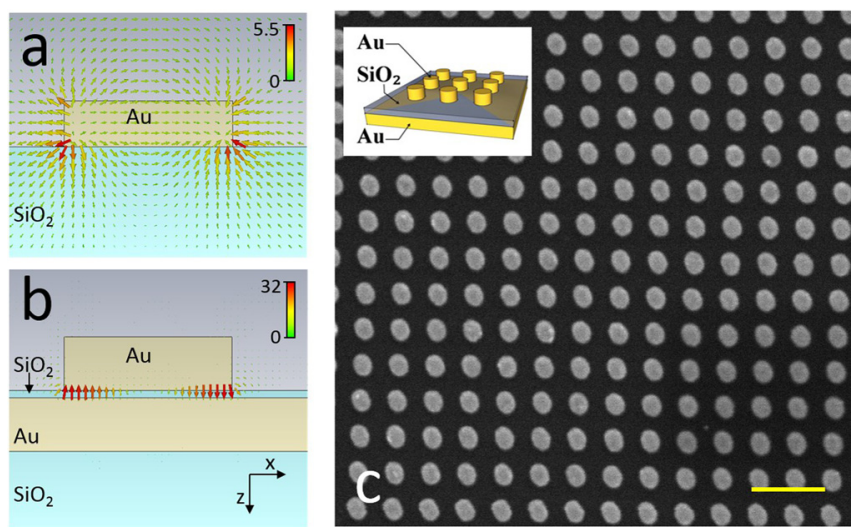

FIG. 1. Resonant local electric field enhancement for $110 \mathrm{~nm}$ diameter gold nanodisks (a) on a glass substrate and (b) in a MDM patch antenna configuration with a $5 \mathrm{~nm} \mathrm{SiO}_{2}$ spacer layer. (c) Scanning electron microscopy image of the Au nanodisk array. Inset: schematic of the MDM array. The scale bar is $1 \mu \mathrm{m}$. 
gaps, we have carried out optical sensing experiments and verified the increased sensitivity of the plasmon resonance peak to the change in the refractive index.

Our samples consist of plasmonic nanodisk arrays fabricated using standard positive resist EBL and subsequent thermal deposition of a $30 \mathrm{~nm}$ gold layer [Fig. 1(c)]. The substrates used for patch antennas were glass cover slips covered with a smooth $30 \mathrm{~nm}$ Au layer and a $5 \mathrm{~nm}$ layer of sputtered $\mathrm{SiO}_{2}$ that acts as a spacer. To produce samples with exposed near fields, the $\mathrm{SiO}_{2}$ layer was selectively etched, resulting in a narrower pedestal under the nanodisks. Inductively Coupled Plasma (ICP) etching with $\mathrm{CF}_{4}$ and $\mathrm{O}_{2}$ gases was used to selectively etch the $\mathrm{SiO}_{2}$ layer in the patch antenna gap, without etching Au. We have also used Indium Tin Oxide (ITO) covered glass substrates to produce control samples without extreme field confinement. Several arrays with varying nanoparticle sizes were fabricated to control the spectral position of the plasmon resonance. The periodicity of arrays was set to $250 \mathrm{~nm}$ or $350 \mathrm{~nm}$; however, no periodicity-dependent effects were observed in our experiments. Optical measurements were carried out in the transmission configuration where a collimated white light from a tungsten lamp illuminated the sample at normal incidence. The transmitted light was collected using a low numerical aperture $(\mathrm{NA}=0.25)$ objective and directed to a spectrometer. Si-based CCD was used to measure spectra in the $400 \mathrm{~nm}-1000 \mathrm{~nm}$ spectral region, and an InGaAs linear array was used for the $800 \mathrm{~nm}-1600 \mathrm{~nm}$ region. The measured transmittance spectra were normalized to spectra transmitted through the substrates alone, without the presence of the nanodisks, and the resulting transmittance ratios were plotted for comparing the performance of different samples. The true transmittance spectra of the arrays can be inferred from these spectra by multiplying them with the corresponding transmittance spectra of the substrates.

Figure 2 shows our experimental transmission data for MDM patch antenna arrays [Fig. 2(a)] and for nanodisks fabricated on ITO-coated glass substrates [Fig. 2(b)]. The latter show a conventional spectral dip characteristic of light extinction due to the plasmonic resonance, whereas the MDM arrays feature dispersive transmission curves resembling Fano resonances. The position of the Fano feature is sensitive to the size of the nanodisks with larger diameter nanoparticles producing red-shifted features [Fig. 2(a)]; however, the experimental and numerically simulated spectra are not sensitive to the periodicity of the arrays (data not shown). The Fano shape of the transmittance spectra can be understood in terms of coupling of the narrow spectral plasmonic resonance to the
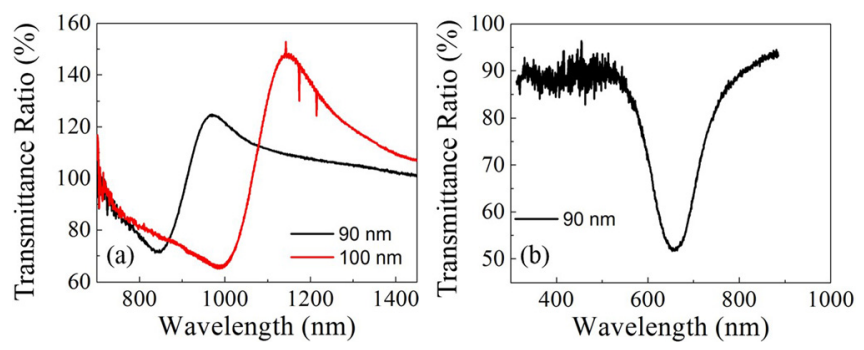

FIG. 2. Transmission spectra of (a) the MDM structures featuring a Fano resonance and (b) an Au nanodisk array on the ITO substrate showing only a Lorentzian dip. continuum of the white light transmitted through the substrate. The interference of the fields scattered by discrete nanoparticles with directly transmitted fields can produce complex lineshapes depending on the relative phase between the scattered and the background optical fields. ${ }^{21,22}$ Note that the transmittance of the bare $30 \mathrm{~nm}$ Au film in the near infrared spectral region is $<10 \%$, and the presence of the nanoparticle scattering can result in enhanced light transmission in the case of constructive interference. The scattered light undergoes the $\pi$ phase shift as the frequency of light is swept through the plasmon resonance, which results in its destructive interference with the driving field to the blue of the resonance and enhanced transmission to the red of the resonance. In the absence of the metal film, nearly all the light is transmitted through the substrate; therefore, the presence of the nanoparticles can only result in decreased transmittance due to absorption and scattering. Unlike the case of the Fano resonances produced by the coupling between the bright and dark plasmonic modes in the nanoparticles, ${ }^{23-27}$ the Fano coupling observed here between the far field excitation and the scattered field results in only modest improvement of the spectral width of the resonance, as we will show below.

To verify the quality of the nanofabricated samples and our interpretation of their experimental transmittance data, we performed numerical simulations using the commercial software CST Microwave Studio. All the geometric parameters in these simulations were taken from the experimental samples. Along the z-axis, the mesh lengths for the simulations were set to be $7 \mathrm{~nm}$ in the metal and $1 \mathrm{~nm}$ in the $\mathrm{SiO}_{2}$ gap, while along the $x-y$ plane, the mesh size was $10 \mathrm{~nm}$. The dielectric constant of the $\mathrm{SiO}_{2}$ substrate and gap was fixed at 2.13. As for the dielectric constant of gold, the Drude model

$$
\epsilon_{A u}=\epsilon_{\infty}-\omega_{p}^{2} / \omega(\omega-i \gamma)
$$

was used to describe its frequency dependence. The parameters $\epsilon_{\infty}=10, \omega_{p}=1.37 \times 10^{16} \mathrm{rad} / \mathrm{s}$, and $\gamma=6.75 \times 10^{14} \mathrm{~Hz}$ were obtained by fitting the Drude model to the experimentally measured permittivity of the bulk Au material.

In the simulations, light was normally incident onto the nanodisk arrays. The far-field transmission spectra were obtained by integrating the Poynting vectors at a plane placed at $800 \mathrm{~nm}$ below the metal film inside the $\mathrm{SiO}_{2}$ substrate. Light transmitting through a $35 \mathrm{~nm}$ bare metal film was employed for transmission normalization. The local field spectra averaged over the nanocavity edges are used to represent the total local field enhancements. With the increasing patch size from $90 \mathrm{~nm}$ to $110 \mathrm{~nm}$, we see that the wavelengths of the resonant MDM cavity modes (local field enhancement spectra) can be shifted from $900 \mathrm{~nm}$ to $1085 \mathrm{~nm}$ as shown in Fig. 3(a) (dashed lines). Correspondingly, the calculated transmittance ratio spectra, shown in Fig. 3(a) (solid lines lines), will experience the same redshift. As it can be seen from these calculations, the local field resonances possess an approximately Lorentzian lineshape, whereas the calculated transmission spectra feature a typical Fano-type resonance. The wavelength of the local field enhancement peak is located between the corresponding transmission dip and the peak. Thus, the transmission dips are caused by the destructive interference between the directly 


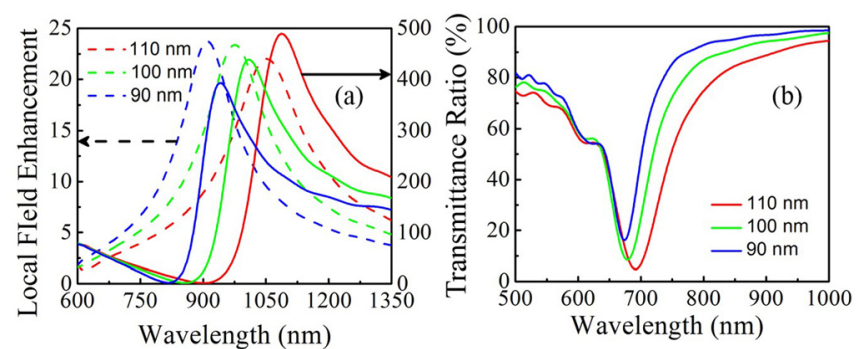

FIG. 3. (a) Calculated transmission spectra normalized by metal film transmission (solid curves) and local field enhancement (dashed curves) for patch nanoantennas for different nanodisk diameters. (b) Calculated transmission spectra of Au nanodisk arrays with different diameters on the ITO-covered glass substrate.

transmitted field and the resonantly transmitted field through the metal film. In contrast, the calculated transmittance for the gold nanodisks on ITO covered substrates features only a dip [Fig. 3(b)], in accordance with our experimental data. It should be noted that the measured transmittance ratios are significantly smaller than the calculated values due to the imperfect interference conditions caused by the finite NA of the collection objective (Fig. S1 in the supplementary material).

Typically, the localized plasmon resonances of nanoparticles depend on not only the size and shape of the particle but also the refractive index of their environment. Thus, by tracking the shifts of plasmonic spectral peaks, one can measure the change in the refractive index of the surrounding medium and use this for particle detection and molecular and chemical sensing. ${ }^{28-31}$ The sensitivity of such refractive index sensors typically depends on two main parameters. First, the width of the optical resonance is important, with narrower peaks producing better sensors. Second, the spectral shift per refractive index unit change determines the sensitivity of the sensor to the change in the environment.

The strong field confinement in the MDM patch antennas makes them attractive for optical sensing since they are expected to be very sensitive to small changes in the refractive index in the gap. However, in our samples shown in Figs. 1(b) and 1(c), the strongly confined near fields are not exposed and thus cannot be used for optical sensing. To expose the near fields of our platform and make them accessible to sense the refractive index of the environment, we have performed isotropic etching of the samples. The resulting structures feature gold nanodisks standing on a $\mathrm{SiO}_{2}$ pedestal on a gold film [Fig. 4(f), inset] exhibiting exposed near fields that are ideal as optical sensors with ultrasmall sensing volumes.

To test the sensitivity of our samples, we use widely accepted sensing Figure of Merit (FoM) definition as ${ }^{32}$

$$
F o M=\frac{\left(\Delta \omega_{\text {shift }} / \Delta n\right)}{\Delta \omega_{\text {width }}},
$$
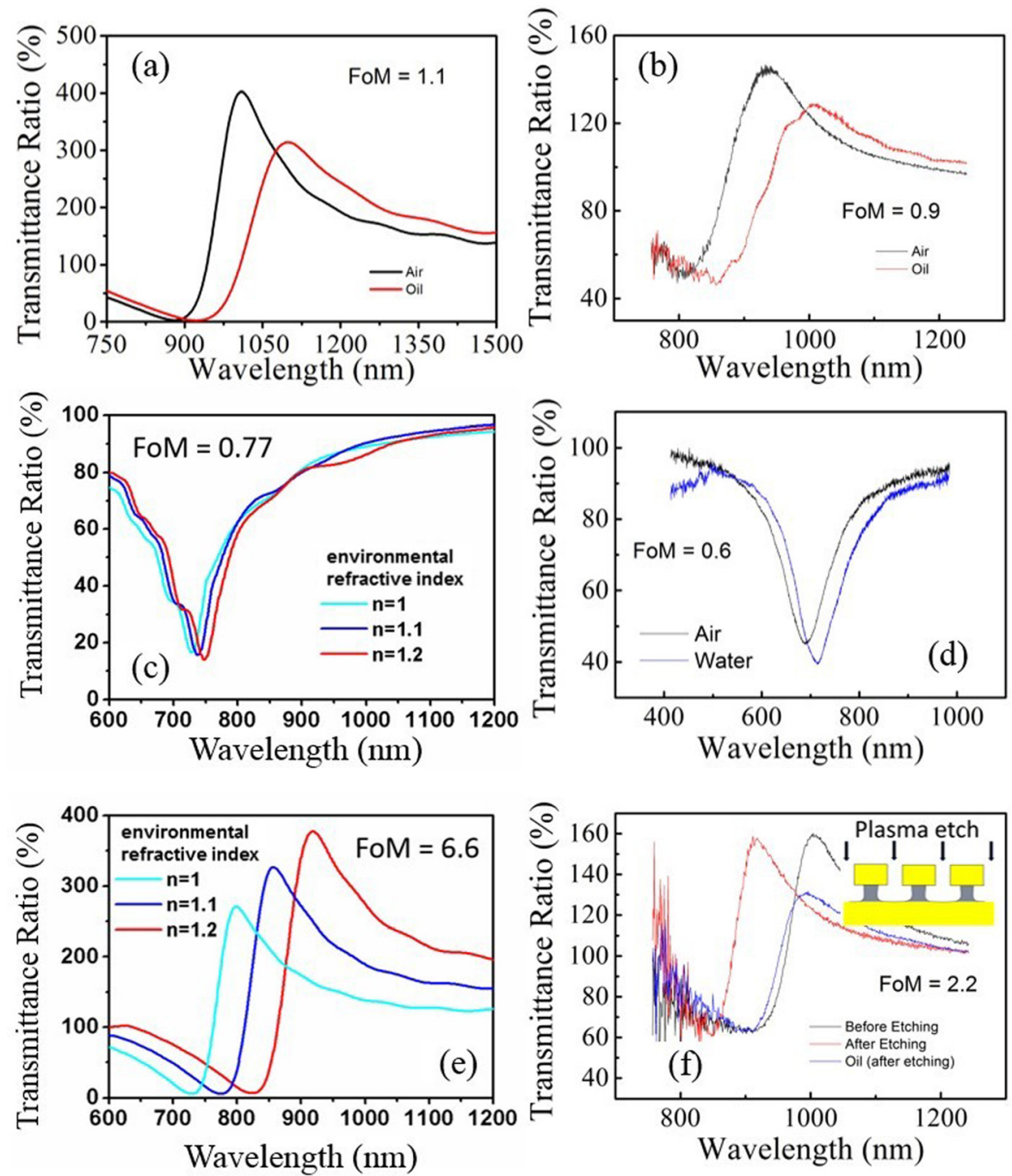

FIG. 4. (a) Theoretical calculated and (b) experimentally measured spectral shifts for unetched MDM patch nanoantennas in different environments. (c) Theoretical calculated and (d) experimentally measured spectral shifts for nanodisks on ITO-covered glass substrates in different environments. (e) Theoretical calculated and (f) experimentally measured spectral shifts for etched MDM patch nanoantennas on a pedestal in different environments. (f) The transmittance of the array before plasma etching (black curve). Inset: schematics of patch antennas on a pedestal. 
where $\Delta \omega_{\text {shift }} / \Delta n$ is the frequency shift per refractive index unit change and $\Delta \omega_{\text {width }}$ is the width of the resonance. For the latter parameter, the full width at half maximum of the peak is used for Lorentzian resonance. For Fano resonances, due to the asymmetric lineshape, we instead use the frequency difference between the Fano dip and its closest peak, as accepted in previous studies. ${ }^{24,25}$

Figure 4 shows the results of our sensing experiments and simulations. For the unetched MDM nanodisk sample, we measure the transmittance ratio of the sample in air and compare it to the plasmon peak shift when it is immersed in oil with a refractive index of 1.51 [Fig. 4(b)]. The experimental FoM for this system exhibits a modest value of $\sim 0.9$ which is in a good agreement with the numerical simulations shown in Fig. 4(a). The nanodisks on the ITO substrate similarly exhibit only a FoM of $\sim 0.7$ [Figs. 4(c) and 4(d)]. For the etched MDM patch antennas, the experimental FoM is calculated by performing a transmittance measurement in air and in immersion oil. Samples with slightly larger gaps $(8 \mathrm{~nm})$ were used in this experiment. A much larger value of FoM $=2.2$ is observed for etched MDM samples [Fig. 4(f)]. It is worth noting that after immersing the sample in oil, the transmittance spectrum resembles the spectrum measured in air before etching. This indicates that the position of the plasmon peak mainly depends on the refractive index of the area where the near fields are concentrated, i.e., in the gap between the nanodisk and the metal film. The determination of FoM of the same sample for media with different refractive indices yields similar results (Fig. S2 in the supplementary material). The theoretical FoM for patch antennas calculated from Fig. 4(e) for a $50 \mathrm{~nm}$ radius $\mathrm{SiO}_{2}$ pedestal is even higher and reaches the value of 6.6. This is comparable to the highest FoM values reported in the literature for isolated particles, ${ }^{24,27,28,33}$ however, our sensor possesses much smaller sensing volume. The relatively smaller experimental value compared to the numerical FoM is possibly due to the fact that the samples were under-etched. Numerical simulations with different undercuts support this view showing deteriorated FoM values in the case of insufficient etching (Fig. S3 in the supplementary material). Thus, the experimental FoM can be improved further by optimizing the etching procedure and by using samples with smaller gaps. It is worth noting that the improvement in the FoM for patch antenna arrays is mostly due to the larger shift per unit refractive index change $\Delta \omega_{\text {shift }} / \Delta n$ and not the significantly smaller width of the resonance $\Delta \omega_{\text {width }}$. For example, in the system shown in Fig. 4 for $\Delta \omega_{\text {width }}$, we have $52.83 \mathrm{THz}$ and $36.74 \mathrm{THz}$, whereas $\Delta \omega_{\text {shift }} / \Delta n$ is $52.35 \mathrm{THz}$ and $238 \mathrm{THz}$ for ITO-covered sample and patch antennas, respectively.

In conclusion, we have developed a platform with highly confined, exposed near fields in the optical regime. Metaldielectric-metal patch nanoantennas are used to produce strongly localized fields in a spacer layer, which are exposed to the environment by selective etching of the dielectric layer. To confirm the performance of our optical platform, we have carried out optical sensing experiments and have demonstrated sensitivity to the refractive index change in ultrasmall volumes. Our approach paves the way for applications, where highly reproducible nanostructures with exposed strongly confined near fields can be beneficial, such as optical detection of molecular layers and bioparticles, coupling plasmonic fields to single photon emitters such as quantum dots, and placing nonlinear nanomaterials in plasmonic hot spots.

See supplementary material for transmittance spectra for different collection NA, FoM determination with different refractive index media, and numerical calculations of different degrees of undercut.

This work was supported by the Emory University startup funds. We thank the University of Georgia and the Georgia Institute of Technology cleanroom facilities for their invaluable help with nanofabrication. S.G.R. acknowledges support from the Spanish Ministry of Science and Innovation under Project No. MAT2014-53432-C5-1-R.

${ }^{1}$ L. Novotny and N. F. van Hulst, “Antennas for light," Nat. Photonics 5, 83-90 (2011).

${ }^{2}$ J. N. Anker, W. P. Hall, O. Lyandres, N. C. Shah, J. Zhao, and R. P. van Duyne, "Biosensing with plasmonic nanosensors," Nat. Mater. 7, 442-453 (2008).

${ }^{3}$ L. Tong, H. Wei, S. Zhang, and H. Xu, "Recent advances in plasmonic sensors," Sensors 14(5), 7959-7973 (2014).

${ }^{4}$ M. Kauranen and A. V. Zayats, "Nonlinear plasmonics," Nat. Photonics 6, 737-748 (2012).

${ }^{5}$ N. P. de Leon, M. D. Lukin, and H. Park, "Quantum plasmonic circuits," IEEE J. Sel. Top. Quantum Electron. 18, 1781-1791 (2012).

${ }^{6}$ P. Mühlschlegel, H.-J. Eisler, O. J. F. Martin, B. Hecht, and D. W. Pohl, "Resonant optical antennas," Science 308, 1607 (2005).

${ }^{7}$ K. J. Savage, M. M. Hawkeye, R. Esteban, A. G. Borisov, J. Aizpurua, and J. J. Baumberg, "Revealing the quantum regime in tunnelling plasmonics," Nature 491, 574-577 (2012).

${ }^{8}$ C. Ciraci, R. Hill, J. J. Mock, A. Dominguez, Y. Urzhumov, S. Maier, J. B. Pendry, A. Chilkoti, and D. R. Smith, "Probing the ultimate limits of plasmonic enhancement," Science 337, 1072 (2012).

${ }^{9}$ W. Zhu, R. Esteban, A. G. Borisov, J. J. Baumberg, P. Nordlander, H. J. Lezec, J. Aizpurua, and K. B. Crozier, "Quantum mechanical effects in plasmonic structures with subnanometre gaps," Nat. Commun. 7, 11495 (2016).

${ }^{10}$ A. Rose, T. B. Hoang, F. McGuire, J. J. Mock, C. Ciraci, D. R. Smith, and M. H. Mikkelsen, "Control of radiative processes using tunable plasmonic nanopatch antennas," Nano Lett. 14, 4797-4802 (2014).

${ }^{11}$ J. Mertens, A. L. Eiden, D. O. Sigle, F. Huang, A. Lombardo, Z. Sun, R. S. Sundaram, A. Colli, C. Tserkezis, J. Aizpurua, S. Milana, A. C. Ferrari, and J. J. Baumberg, "Controlling sub-nm gaps in plasmonic dimers using graphene," Nano Lett. 13, 5033 (2013).

${ }^{12}$ H. Harutyunyan, A. B. F. Martinson, D. Rosenmann, L. K. Khorashad, L. V. Besteiro, A. O. Govorov, and G. P. Wiederrecht, "Anomalous ultrafast dynamics of hot plasmonic electrons in nanostructures with hot spots," Nat. Nanotechnol. 10, 770-774 (2015).

${ }^{13}$ S. Huang, T. Ming, Y. Lin, X. Ling, Q. Ruan, T. Palacios, J. Wang, M. Dresselhaus, and J. Kong, "Ultrasmall mode volumes in plasmonic cavities of nanoparticle-on-mirror structures," Small 12(37), 5190-5199 (2016).

${ }^{14}$ N. Liu, M. Mesch, T. Weiss, M. Hentschel, and H. Giessen, "Infrared perfect absorber and its application as plasmonic sensor," Nano Lett. 10(7), 2342-2348 (2010).

${ }^{15}$ G. Hajisalem, M. S. Nezami, and R. Gordon, "Probing the quantum tunneling limit of plasmonic enhancement by third harmonic generation," Nano Lett. 14(11), 6651-6654 (2014).

${ }^{16}$ J. B. Lassiter, X. Chen, X. Liu, C. Ciraci, T. Hoang, S. Larouche, S.-H. Oh, M. H. Mikkelsen, and D. Smith, "Third-harmonic generation enhancement by film-coupled plasmonic stripe resonators," ACS Photonics 1, 1212-1217 (2014).

${ }^{17}$ S. Shen, L. Meng, Y. Zhang, J. Han, Z. Ma, S. Hu, Y. He, J. Li, B. Ren, T.-M. Shih, Z. Wang, Z. Yang, and Z. Tian, "Plasmon-enhanced secondharmonic generation nanorulers with ultrahigh sensitivities," Nano Lett. 15(10), 6716-6721 (2015).

${ }^{18}$ T. B. Hoang, G. M. Akselrod, and M. H. Mikkelsen, "Ultrafast roomtemperature single photon emission from quantum dots coupled to plasmonic nanocavities," Nano Lett. 16(1), 270-275 (2016). 
${ }^{19}$ F. Wang, A. B. F. Martinson, and H. Harutyunyan, "Efficient nonlinear metasurface based on nonplanar plasmonic nanocavities," ACS Photonics 4(5), 1188-1194 (2017).

${ }^{20}$ E.-M. Roller, C. Argyropoulos, A. Hoegele, T. Liedl, and M. Pilo-Pais, "Plasmon-exciton coupling using DNA templates," Nano Lett. 16(9), 5962-5966 (2016).

${ }^{21}$ R. Nicolas, G. Leveque, J. Marae-Djouda, G. Montay, Y. Madi, J. Plain, Z. Herro, M. Kazan, P.-M. Adam, and T. Maurer, "Plasmonic mode interferences and Fano resonances in metal-insulator- metal nanostructured interface," Sci. Rep. 5, 14419 (2015).

${ }^{22}$ M. Svedendahl and M. Kall, "Fano interference between localized plasmons and interface reflections,” ACS Nano 6(8), 7533-7539 (2012). PMID: 22808902.

${ }^{23}$ F. Hao, Y. Sonnefraud, P. V. Dorpe, S. A. Maier, N. J. Halas, and P. Nordlander, "Symmetry breaking in plasmonic nanocavities: Subradiant LSPR sensing and a tunable Fano resonance," Nano Lett. 8, 3983-3988 (2008).

${ }^{24}$ Y. Zhan, D. Y. Lei, X. Li, and S. A. Maier, "Plasmonic Fano resonances in nanohole quadrumers for ultra-sensitive refractive index sensing," Nanoscale 6, 4705-4715 (2014).

${ }^{25}$ J. A. Fan, K. Bao, C. Wu, J. Bao, R. Bardhan, N. J. Halas, V. N. Manoharan, G. Shvets, P. Nordlander, and F. Capasso, "Fano-like interference in self-assembled plasmonic quadrumer clusters," Nano Lett. 10, 4680-4685 (2010).
${ }^{26}$ A. A. Yanik, A. E. Cetin, M. Huang, A. Artar, S. H. Mousavi, A. Khanikaev, J. H. Connor, G. Shvets, and H. Altug, "Seeing protein monolayers with naked eye through plasmonic Fano resonances," Proc. Natl. Acad. Sci. U.S.A. 108, 11784-11789 (2011).

${ }^{27}$ J. B. Lassiter, H. Sobhani, J. A. Fan, J. Kundu, F. Capasso, P. Nordlander, and N. J. Halas, "Fano resonances in plasmonic nanoclusters: Geometrical and chemical tunability," Nano Lett. 10, 3184-3189 (2010).

${ }^{28}$ K. M. Mayer and J. H. Hafner, "Localized surface plasmon resonance sensors," Chem. Rev. 111, 3828-3857 (2011).

${ }^{29}$ A. A. Yanik, M. Huang, O. Kamohara, A. Artar, T. W. Geisbert, J. H. Connor, and H. Altug, "An optofluidic nanoplasmonic biosensor for direct detection of live viruses from biological media,” Nano Lett. 10(12), 4962-4969 (2010).

${ }^{30}$ F. V. Ignatovich and L. Novotny, "Real-time and background-free detection of nanoscale particles," Phys. Rev. Lett. 96(1), 013901 (2006).

${ }^{31}$ N. Liu, M. L. Tang, M. Hentschel, H. Giessen, and A. P. Alivisatos, "Nanoantenna-enhanced gas sensing in a single tailored nanofocus," Nat. Mater. 10, 631-636 (2011).

${ }^{32}$ L. J. Sherry, S.-H. Chang, G. C. Schatz, R. P. Van Duyne, B. J. Wiley, and Y. Xia, "Localized surface plasmon resonance spectroscopy of single silver nanocubes," Nano Lett. 5, 2034-2038 (2005).

${ }^{33}$ N. Verellen, P. Van Dorpe, C. Huang, K. Lodewijks, G. A. E. Vandenbosch, L. Lagae, and V. V. Moshchalkov, "Plasmon line shaping using nanocrosses for high sensitivity localized surface plasmon resonance sensing," Nano Lett. 11, 391-397 (2011). 